\title{
LA CULTURA DE PAZ Y CONFLICTOS: IMPLICACIONES SOCIOEDUCATIVAS
}

\author{
THE CULTURE OF PEACE AND CONFLICTS: \\ SOCIO-EDUCATIONAL IMPLICATIONS
}

\section{SEBASTIÁN SÁNCHEZ FERNÁNDEZ* VICTORIA PÉREZ DE GUZMÁN** TERESA REBOLLEDO GÁMEZ*** ROCÍO RODRÍGUEZ CASADO****}

\section{RESUMEN}

El conocimiento y el tratamiento educativo de las manifestaciones de la Cultura de Paz constituyen un principio fundamental para prevenir cualquier tipo de violencia, visibilizando la presencia en nuestras vidas de múltiples situaciones pacíficas que nos permiten gestionar los conflictos de modo no violento. Realizando una revisión sobre los hitos de la Cultura de Paz a nivel internacional y sus implicaciones en el ámbito educativo, concluimos con diversas estrategias para fomentar valores, actitudes, comportamientos y estilos de vida que permiten resolver conflictos por medios pacíficos, promoviendo, entre otros, la solidaridad, respeto, diálogo, justicia y, especialmente, la igualdad entre mujeres y hombres.

PALABRAS CLAVES: cultura de paz, conflicto, educación.

\section{ABSTRACT}

The knowledge and the educational treatment of the manifestations of the Culture of Peace constitute a fundamental beginning to anticipate any type of violence, visualizing the presence in our lives of multiple pacific situations that allow us to manage the conflicts of way no violent. Conducting a review on the milestones of the Culture of Peace at the international level and its implications in the educational field, we conclude with strategies to promote values, attitudes, behaviors and lifestyles that allow resolving conflicts of peaceful means, promoting, among others, solidarity, respect, dialogue, justice and, especially, equality between women and men.

KEYWORDS: culture of peace, conflict, education.

\footnotetext{
* Doctor en Ciencias de la Educación. Catedrático de Didáctica en la Universidad de Granada. Facultad de Educación, Campus de la Universidad de Granada en Melilla, Calle Santander 1, CP 52071, Melilla-España. Correo electrónico: ssanchez@ugr.es

** Doctora en Pedagogía. Profesora titular en la Universidad Pablo de Olavide. Universidad Pablo de Olavide, Facultad de Ciencias Sociales, Carretera Utrera, km. 1, CP 41013 Sevilla. Correo electrónico: mvperpuy@upo.es

*** Doctora en Desarrollo y Ciudadanía. Profesora Asociada en la Universidad Pablo de Olavide. Facultad de Ciencias Sociales, Carretera Utrera, km. 1, CP 41013 Sevilla. Correo electrónico: tjrebgam@upo.es

**** Doctora en Desarrollo y Ciudadanía. Profesora sustituta interina Universidad Pablo de Olavide. Utrera, km. 1, CP 41013 Sevilla. Correo electrónico: mrrodcas@upo.es
} 


\section{HITOS SIGNIFICATIVOS Y CONCEP- TO DE CULTURA DE PAZ}

La primera definición de Cultura de Paz se realizó en el Congreso Internacional de Yamoussoukro (Costa de Marfil) en 1989 y fue adoptada como Programa de la UNESCO en 1995, tal y como se desarrolló después en una publicación de la propia organización (UNESCO, 1996).

En la Resolución 53.243.de la Declaración y Programa de Acción sobre una Cultura de Paz, aprobada por la Asamblea General de Naciones Unidas el 13 de septiembre de 1999, define la Cultura de Paz como un conjunto de valores, actitudes, tradiciones, comportamientos y estilos de vida basados en una serie de principios y compromisos (Art. 1), y en los dos artículos siguientes, se reconoce expresamente que la educación a todos los niveles es uno de los medios fundamentales para edificar una cultura de paz (Art. 4). Dentro de este Programa de Acción, se especifican varias medidas para dar a conocer, extender y consolidar la Cultura de Paz (Art.9.B):

$\diamond \quad$ Promover una cultura de paz por medio de la educación. Cabe destacar dentro de esta medida el velar porque las personas desde su infancia reciban instrucción sobre valores, comportamientos, actitudes y estilos de vida que les ayuden a resolver los conflictos a través de medios pacíficos. De igual modo, se hace hincapié en velar porque hay igualdad de acceso de las mujeres a la educación, especialmente de las niñas.

$\diamond$ Promover el desarrollo económico y social sostenible. En este apartado se recogen varios aspectos, de los que se destacan: incluir la perspectiva de género y el fomento de la autonomía de mujeres y niñas como parte integrante del proceso de desarrollo, tomar medidas especiales que atiendan a las necesidades de mujeres y niños y a grupos con necesidades especiales $\mathrm{y}$, reforzar los procesos de rehabilitación, reintegración y reconciliación de todas las personas involucradas en un conflicto.

$\diamond$ Promover el respeto de todos los derechos humanos. Con objeto de difundir y promover la Declaración Universal de Derechos Humanos a todos los niveles.

$\diamond$ Garantizar la igualdad entre mujeres y hombres. Cabe destacar el integrar la perspectiva de género en la aplicación de todos los instrumentos internacionales pertinentes y promover la igualdad entre hombres y mujeres en la adopción de decisones sociales, políticas y económicas.

$\diamond$ Promover la participación democrática. Afianzar actividades para promver principios y prácticas democráticos en todos los niveles de la enseñanza escolar, extraescolar y no escolar y afianzarlos

$\diamond$ Promover la comprensión, la tolerancia y la 
solidaridad. Entre otras, tomar medidas que promuevan la comprensión, la tolerancia y la solidaridad en toda la sociedad, especialmente con los grupos vulnerables. Así como, fomentar la cooperación entre los pueblos y entre las naciones y dentro de ellas.

$\diamond$ Apoyar la comunicación participativa y libre circulación de información y conocimientos. Se debe apoyar a los medios de difusión que promueven la cultura de paz y fomentar la comunicación social con el objetivo de que las comunidades puedan expresar sus necesidades y participar en la adopción de decisiones.

$\diamond \quad$ Promover la paz y seguridad internacionales. Destacar la necesidad de trabajar en pro de una paz justa y duradera en todas partes del mundo y apoyar actividades, a todos los niveles, que traten de solucionar problemas concretos producidos tras las conflictos.

Especial relevancia adquiere el manifiesto denominado "Manifiesto 2000 para una cultura de paz y de no violencia”, redactado en París en marzo de 1999, por un grupo de premios Nobel de la Paz', con motivo del quinquagésimo aniversario de la Declaración Universal de los Derechos Humanos. Se invita a todo individuo a pensar en su responsabilidad en

1 Los catorce Premios Nobel que han sido los impulsores y primeros signatarios del Manifiesto son: Norman Borlaug, Adolfo Pérez Esquivel; Dalai Lama, MikhaII Sergeyevich Gorbachov; Mairead Maguire; Nelson Mandela; Rigoberta Menchu Turn, Shimon Peres; Jose Ramos Horta,- Joseph Roblat, Desmond Mpilo Tutu; David Trimble, Elie Wiesel, Carlos Felipo Ximenes Belo. el fomento de la cultura de la paz. Recoge de forma resumida, en un lenguaje sencillo, los seis principios claves que la definen y la determinan. El manifiesto dice así:

Reconociendo mi parte de responsabilidad ante el futuro de la humanidad, especialmente para los niños de hoy y de mañana, me comprometo en mi vida diaria, en mi familia, mi trabajo, mi comunidad, mi país y mi región a:

- respetar la vida y la dignidad de cada persona, sin dicriminación ni prejuicios;

- practicar la no violencia activa, rechazando la violencia en todas sus formas: física, sexual, psicológica, económica y social, en particular hacia los más débiles y vulnerables, como los niños y los adolescentes;

- compartir mi tiempo y mis recursos materiales cultivando la generosidad a fin de terminar con la exclusión, la injusticia y la opresión política y económica;

- defender la libertad de expresión y la diversidad cultural privilegiando siempre la escucha y el diálogo, sin ceder al fanatismo, ni a la maledicencia y el rechazo del prójimo;

- promover un consumo responsable y un modo de desarrollo que tenga en cuenta la importancia de todas las formas de vida y el equilibrio de los recursos naturales del planetay

- contribuir al desarrollo de mi comunidad, propiciando la plena participación de las mujeres y el respeto de los principios de- 
mocráticos, con el fin de crear juntos nuevas formas de solidaridad.

Según se indica en el manifiesto, es la responsabilidad de cada ser humano de convertir en realidad los valores, las actitudes, los comportamientos que fomentan la cultura de paz. Porque cada uno puede actuar en el marco de su familia, su localidad, su ciudad, su región, y su país practicando y fomentando la no violencia, la tolerancia, el dialogo, la reconciliación, la justicia y la solidaridad día a día.

Según estos pronunciamientos, a partir del año 2000 se daría un nuevo comienzo para transformar la cultura de guerra y de violencia en una cultura de paz y de no violencia. Esta evolución exige la participación de todas las personas y les da a la juventud y a las generaciones futuras valores que les ayuden a forjar un mundo más digno y armonioso, un mundo de justicia, solidaridad, libertad y prosperidad. La cultura de paz hace posible el desarrollo duradero, la protección del medio ambiente y la satisfacción personal de cada ser humano.

Todo este movimiento de cultura de paz a nivel mundial promovió que Naciones Unidas proclamara el año 2000 como Año Internacional de la Cultura de la Paz, al igual que la primera década del siglo XXI como Decenio Internacional de la promoción de una Cultura de Paz y Noviolencia en beneficio de los niños y niñas del mundo (2001-2010). La UNESCO coordina las actividades del Año Internacional de la Cultura de Paz en el mundo entero, tanto en la enseñanza reglada como no reglada, potenciando políticas y principios de acción favorables para una ciudadanía democrática y el fomento de los derechos humanos.

Por otro lado, los 17 Objetivos de Desarrollo Sostenible (ODS), fueron redactados en septiembre de 2015 con objeto de erradicar la pobreza, proteger el planeta y asegurar la prosperidad de todos como parte de una nueva agenda de desarrollo sostenible. El objetivo número 16 está centrado en promover sociedades justas, pacíficas e inclusivas. Dentro de sus metas, cabe destacar: Reducir significativamente todas las formas de violencia y las correspondientes tasas de mortalidad en todo el mundo, Poner fin al maltrato, la explotación, la trata y todas las formas de violencia y tortura contra los niños, Garantizar la adopción en todos los niveles de decisiones inclusivas, participativas y representativas que respondan a las necesidades y Promover y aplicar leyes y políticas no discriminatorias en favor del desarrollo sostenible.

En la XVI Cumbre Mundial de Premios Nobeles de la Paz (2017) fue escenario para la redacción de los diez principios para la paz propuestos en la "Carta de Colombia":

1. La paz es un derecho. Es el derecho de nacimiento de cada persona y el derecho supremo de la humanidad.

2. Somos uno. La Humanidad es una sola fa- 
milia y compartimos el don de la vida sobre este frágil planeta. Lo que le pasa a uno, nos pasa a todos.

3. Somos diversos. La diversidad enriquece a nuestra humanidad. Es un patrimonio que debemos honrar y cuidar.

4. Debemos seguir la regla de oro. El principio moral de tratar a los otros como queremos ser tratados nosotros mismos debe ser aplicado no solo a la conducta de las personas sino también a la conducta de las religiones y naciones.

5. Debemos evitar la guerra. La guerra destruye el tejido humano y representa un fracaso para la humanidad.

6. Debemos ser legales y justos. La paz y la estabilidad del mundo requieren la adhesión y el respeto al Derecho Internacional, incluyendo las normas internacionales de los Derechos Humanos y el Derecho Internacional Humanitario. Una paz duradera solo puede alcanzarse si se basa en la justicia social.

7. Debemos hablar. Siempre que sea posible, los conflictos deben terminar mediante el diálogo. La comunidad internacional debe apoyar medidas efectivas para prevenir y limitar las guerras.

8. Debemos respetarnos. Aun dentro del conflicto, debemos reconocer a todo enemigo como un ser humano que merece respeto, cuyas motivaciones deben ser entendidas. La eliminación o humillación del adversario es la semilla de más violencia en el futuro.

9. Debemos educar. Hay que promover la tolerancia, la solidaridad, la compasión, el respeto a las diferencias y a los derechos de las minorías, para crear una cultura global de paz. Una educación basada en estos valores debe implementarse en todo el mundo.

10. Debemos entender. Todas las vidas son tan valiosas como nuestra propia vida. Si entendemos esto con nuestros corazones y nuestras mentes, podremos construir y mantener la paz en el mundo para nosotros, para nuestros hijos y las generaciones por venir.

Estos hitos señalados nos hacen reflexionar, una vez más, que la paz, es algo más que la ausencia de guerra. Debemos que ir cambiando la cultura de violencia que existe y se está integrando en los procesos de socialización de los sujetos. Si queremos llegar a una cultura de paz real, debemos cambiar la forma de interpretar las relaciones y de resolver los conflictos que existen. La guerra la justifica quien se cree en posesión de la única verdad. Si bien, nuestro mundo es complejo y en él intervienen cada vez más factores. Se hace necesaria una cultura de paz; si bien, hay que llegar a la comprensión de la diversidad, la interdependencia que existe en todos los niveles, la multicausalidad de los fenómenos sociales, etc. 


\section{LOS CONFLICTOS DESDE LA PERS- PECTIVA DE LA CULTURA DE PAZ}

Desde la Investigación para la Cultura de Paz se nos presenta el conflicto interpersonal y grupal como algo consustancial a la vida, lo que nos lleva a afirmar que donde hay vida hay conflicto. Se vienen estudiando los tipos de conflicto, sus orígenes, manifestaciones y los factores influyentes, observándose que en ocasiones producen situaciones dolorosas y violentas, pero que, como afirman Molina y Muñoz (2004), también en otros muchos casos se resuelven y se gestionan de forma no violenta.

El giro que dio la investigación para la paz y los conflictos especialmente a partir de los años 70 del siglo pasado, supone una de las bases teóricas fundamentales para la comprensión de las situaciones de convivencia intercultural. Entender que los conflictos son inherentes a las relaciones humanas es una base del concepto de paz imperfecta (Muñoz, 2001), como categoría analítica que nos permite: "Romper con las concepciones de la paz como algo perfecto, infalible, utópico, terminado, lejano, inalcanzable; reconocer las prácticas pacíficas allá donde ocurran; pensar la paz como un proceso inacabado que se reconoce y se construye cotidianamente". Trifu (2018) realiza una reflexión hacia la integración del pensamiento de paz en cuanto a las dinámicas positivas de paz. Distingue entre paz estructural y paz positiva y busca puntos de unión entre la paz positiva y la paz imperfecta.

Esta concepción de la paz pone de manifiesto, entre otras cuestiones, la importancia de ser conscientes de la presencia en nuestras vidas de múltiples situaciones pacíficas que nos permiten resolver, regular y transformar los conflictos de modo no violento, así como la necesidad de seguir construyendo espacios y situaciones de paz, aunque existan manifestaciones de la violencia, por muy dolorosas que éstas sean.

Si esperamos a que desaparezcan todas las manifestaciones de la violencia para trabajar y desarrollar situaciones pacíficas, difícilmente lo vamos a conseguir, puesto que las relaciones entre individuos y grupos no se suelen caracterizar por la perfección. En cambio, si asumimos las imperfecciones, los problemas y los conflictos, intentando superarlos y/o resolverlos, a la vez que aprendemos de nuestras experiencias al respecto, iremos avanzando también en la construcción de redes entre realidades y situaciones pacíficas, imperfectas, pero cada vez menos violentas.

Entender el conflicto como contraposición de intereses y/o percepciones es una de las formulaciones más usadas y resulta muy útil para comenzar a hablar de ellos, aunque haya que hacer algunas precisiones. Esta noción de conflicto abre grandes posibilidades de análisis por su relación con las necesidades, los 
deseos, las emociones, y otras circunstancias personales y grupales que forman parte de todo el entramado social. En consecuencia, la especie humana podemos definirla como conflictiva en la medida en que tales contraposiciones están siempre presentes en todas las sociedades y actividades humanas (Muñoz, 2004).

Aunque ahora está completamente asumido el descubrimiento de los intereses como características fundamentales de los conflictos, tal idea supuso toda una renovación teórica. En la actualidad también se distinguen necesidades, objetivos e intereses, que aunque pueden ser coincidentes también pueden ser cosas distintas, pero que en definitiva incluyen aquellos presupuestos materiales, espirituales o sociales que las entidades humanas consumen, usan, utilizan, etc., para alcanzar sus expectativas de realización como seres humanos y, que en esta medida, son fundamentales, indispensables o imprescindibles, y su carencia causa desequilibrios y tensiones.

El contenido de estas necesidades ha variado conforme lo ha hecho la propia entidad humana. Cabe preguntarse qué condiciones son imprescindibles para que cualquier grupo humano consiga vivir dignamente. De hecho, existen diversas teorías sobre las necesidades humanas que hacen más o menos hincapié en unas u otras, algunas de ellas distinguen entre necesidades humanas básicas o primarias, otras entre materiales, emocionales o socia- les, etc., pero en cualquier caso todas buscan su validez para todas las culturas, su universalidad.

Poner el enfoque en la satisfacción de las necesidades humanas resulta útil como marco analítico de los conflictos, ya que prioriza el estudio de las fuentes de la conflictividad y las raíces de los problemas para de esta forma evitar o prevenir la violencia. Pero, obviamente, la percepción de los posibles conflictos está conformada por la cultura, la educación $\mathrm{y}$ las influencias sociales, que juegan un papel clave a la hora de determinar las prioridades e incompatibilidades entre necesidades no satisfechas en un conflicto, que pueden derivar en violencia (Sánchez-Vázquez, 2014).

Las percepciones son un mecanismo por el cual recibimos la información sobre las circunstancias que rodean a un conflicto y que, por lo tanto, nos ayudan a calificar y clarificar ante qué situación nos encontramos. No todas las percepciones tienen el mismo carácter y un mismo actor, puede tener diferentes percepciones sobre el mismo conflicto, a veces incluso contradictorias. Pueden ser subjetivas, intuitivas, emocionales, razonadas, más o menos objetivas y compartidas, total o parcialmente, con otros actores.

En esta línea perceptiva y de conciencia también deberíamos de distinguir entre las posiciones que adoptan los participantes implicados, es decir qué es lo que explícitamente demandan, y qué otros supuestos y metas hay detrás de 
ellas. Esto hace que a veces las posiciones aparezcan como irreconciliables, pero los intereses o metas no. Y en su caso, los conflictos relacionados con valores (Segura, Alemany y Gallardo, 2016) tienen mayores dificultades de gestión y regulación porque se presenten con mayores dificultades de cambio o como no negociables.

La conciencia que se tenga de un conflicto, de sus causas, de los sistemas y estructuras que lo hacen posible no siempre está elaborada de una manera congruente, nítida o racional. De esta manera podrían existir diversos grados de conciencia: la no percepción de que el conflicto existe, la percepción del mismo pero relacionándolo con causas aleatorias o fuera del control de los humanos (la mala suerte, los demonios, los dioses, $\mathrm{u}$ otras fuerzas sobrenaturales), y por último tener cierta conciencia de las circunstancias o causas que lo provocan.

En definitiva, en el conflicto confluyen tantas circunstancias que debe ser entendido como un proceso multifactorial en el que están presentes muchas de las condiciones que acompañan a los humanos y que llevan implícitas posibilidades dinámicas en diversos sentidos dependiendo de las opciones que se tomen. Como nos dice Sánchez-Vázquez (2014), en determinados momentos, frente a las propuestas pacíficas, aparecen otras tendencias destructivas y violentas. Es decir, frente a algunos conflictos, en vez de optar por una ges- tión altruista y cooperativa, para favorecer al máximo posible al conjunto del grupo, se opta por soluciones egoístas o que favorecen asimétricamente a una parte. Las formas de la violencia son múltiples y las podemos reconocer en las guerras, el armamentismo, los crímenes, la pobreza, el hambre, la incomunicación, el egoísmo, el racismo, la xenofobia, etc. Igualmente podemos saber que unas y otras establecen vínculos y tienden a potenciarse (Segura, 2010).

Normalmente se habla de una escalada de los conflictos cuando éstos crecen en virulencia, entendida como una tendencia a producir una expansión en la que crecen el tamaño y el número de motivos, los precedentes reconocidos, las normas y conductas afectadas, la intensidad de las actitudes negativas y otras circunstancias tales como las estrategias del poder, las tácticas de amenazas, coerciones, y la ansiedad. La escalada tiene relación con los procesos competitivos, de percepciones equivocadas y de pérdida de compromiso con salidas acordadas o pactadas.

Finalmente, unas y otras circunstancias negativas se podrían reforzar, crecer en tamaño y perder parte de sus vínculos con las causas iniciales. Cuando sospechamos que un conflicto puede tener una tendencia violenta y destructiva, también podemos conseguir que gire hacia la creatividad, hacia lo constructivo. Retomando las bases de los conflictos creativos se pueden sugerir escenarios para 
que esto ocurra (Sánchez-Vázquez, 2014):

- Reconocimiento del problema antes de que se degrade

- Concentrar esfuerzos en una regulación pacífica.

- Reconocer la posible disconformidad y frustración si el conflicto se degradase.

- Percepción desde diferentes perspectivas y reformulación de nuevas orientaciones.

- Presentar las alternativas creativas y cooperativas.

- Establecer la comunicación más clara entre las partes.

- Potenciar la motivación positiva; dotarse de un ambiente positivo.

- Tener flexibilidad para acoger nuevas ideas.

Como indica Ruiz (2015), la motivación basada en valores cooperativos y pacíficos puede terminar siendo un punto esencial que pueda movilizar a los demás.

Existen conflictos violentos que pueden permanecer latentes, ocultos por otros de mayores dimensiones, o porque una de las partes tiene tanto poder que no deja posibilidad de que este aparezca. Es normal que esta situación vaya acompañada de diversas formas de resistencia que tienen un potencial peligroso de generación de nuevas formas de violencia. En cualquier caso, existe también un proceso evaluativo por el cual se decide cuándo una actuación es paz o violencia. Aunque parezca extraño, una misma acción puede ser considerada en algún momento como violencia y en otro no. Esta evaluación depende especialmente de los valores, que en definitiva nos aconsejan si dentro de las posibilidades que tenemos en cada momento satisfacemos al máximo las necesidades de las personas y los grupos implicados.

Como indica Lederach (2003), la diferenciación entre episodio y epicentro consiste en que debajo de los episodios subyacen los grandes temas pendientes que requieren ser transformados, como la exclusión, la extrema desigualdad, la violación de los derechos humanos, el desigual acceso a recursos y la débil institucionalidad (Carmona, 2016). A veces entendidas como el "contenido" o la "sustancia" y las "causas originarias", las dimensiones estructurales centran su atención en las áreas relacionadas con las necesidades humanas básicas, la reproducción simple o ampliada, el acceso a recursos y modelos institucionales de toma de decisiones, el reconocimiento o la negación de las identidades y demandas culturales.

Está claro que el conflicto es un concepto clave en la Investigación para la Paz (Binaburo y Muñoz, 2007) y que existe un gran consenso en considerarlo indispensable para entender las realidades pacíficas, que estamos acostumbrados a percibirlas cargadas de pasividad y de determinismo externo (dependen de lo que hagan otros, especialmente los 
más poderosos, incluso de factores ajenos e incontrolables por los seres humanos, divinidades, etc). Los estudios sobre las realidades pacíficas nos afirman que no son perfectas, que dependen de lo que hagamos cada uno de nosotros, que donde hay vida hay conflicto, y que la diversidad añade complejidad y riqueza, aunque también conflictividad, por lo que aprender a convivir con los conflictos se convierte en una necesidad. De hecho, lo hacemos sin ser conscientes de ello en múltiples situaciones de nuestra vida, tanto en el ámbito privado como público. Por ello, una adecuada gestión educativa, social y política de los conflictos se convierte en una rica experiencia de aprendizaje (Mazorra, 2008).

\section{IMPLICACIONES SOCIOEDUCATI- VAS DE LA CULTURA DE PAZ}

Una de las finalidades más importantes asumidas en las investigaciones y los estudios sobre y para la paz es favorecer la visibilización de los comportamientos pacíficos y no violentos de las personas y de los grupos humanos en los diferentes contextos y ubicaciones en que se producen. Este objetivo adquiere especial relevancia en el caso de la educación, ya que mostrar y reconocer las manifestaciones de la paz en la vida diaria se podría convertir, con relativa facilidad, en una base de conocimiento pedagógico capaz de generar estrategias y recursos didácticos para la formación de los estudiantes y de todos los miembros de las comunidades educativas en los valores de la Cultura de Paz en el ámbito escolar y en la sociedad (Sánchez, 2017).

Por ello, el conocimiento y el tratamiento educativo de las manifestaciones de la Cultura de Paz constituyen un principio fundamental para prevenir cualquier tipo de violencia, visibilizando la presencia en nuestras vidas de múltiples situaciones pacíficas que nos permiten gestionar los conflictos de modo no violento, capacitándonos para seguir construyendo espacios y situaciones de convivencia pacífica no exenta de conflictos, incluso cuando existan casos de violencia. Como indican Pérez Serrano y Pérez de Guzmán (2011), muchos problemas que se producen en la convivencia, en las relaciones interpersonales se producen por los estilos de vida que son excesivamente individualistas. La figura "del otro" se ve como un recurso a mi disposición o como una amenaza a la estabilidad y seguridad personal. El racismo y la xenofobia son problemáticas sociales que propician la aparición de conflictos.

Muchas veces no sabemos descubrir las manifestaciones de la Cultura de Paz en las situaciones en que éstas se producen. No es que no se den experiencias de convivencia pacífica, de solidaridad, de amistad, de colaboración, de regulación pacífica de los conflictos, etc. entre los seres humanos. Estos acontecimientos se producen con mucha frecuencia en nuestras 
conductas habituales, incluso en los entornos más próximos (relaciones familiares, profesionales, escolares, de ocio, etc.), siendo mucho más frecuentes que los comportamientos violentos, pero no estamos acostumbrados a detectarlos y menos a enseñarlos y divulgarlos (Sánchez, 2017).

Relacionándolo con la diversidad cultural y su tratamiento educativo, vemos la necesidad de utilizar educativamente la heterogeneidad, particularmente la étnica y cultural, presente en los centros escolares como fuente de experiencias valiosas de aprendizajes. En muchos casos esta diversidad puede percibirse como fuente de problemas, aunque sean más las ocasiones en que suponga un enriquecimiento que las instituciones educativas deben aprovechar. No podemos permitirnos el lujo de despreciar el enriquecimiento que para todos nosotros representa la variedad de culturas y de sus manifestaciones que cada vez está más presente en las aulas.

Como hemos visto, el cuarto principio del $\mathrm{Ma}$ nifiesto 2000 recoge expresamente la diversidad cultural, relacionándola con la escucha activa, que resulta decisiva para dialogar, sobre todo ante personas y realidades diversas y complejas, con diferentes tradiciones, formas de vida, valores y cosmologías que inspiran sus vidas. Igualmente, el segundo y el sexto abogan por rechazar la violencia contra las mujeres y por favorecer su participación en la vida comunitaria. El diálogo implica la tolerancia activa, el respeto y la aceptación y valoración de las diferencias como componentes imprescindibles para la compresión mutua capaz de generar empatía entre los seres humanos para facilitar acciones de colaboración y participación ciudadana responsable (Tuvilla, 2004).

El respeto a la diversidad como uno de los fundamentos de la Cultura de Paz supone favorecer el diálogo, partiendo de la escucha activa y la empatía como actitudes básicas para gestionar adecuadamente las diferencias, especialmente las que pudieran convertirse en conflictivas, en la seguridad de que la valoración respetuosa, aunque no exenta de crítica, va a llevar a la formación de valores de pertenencia a formas de ciudadanía avanzada, superadora de relaciones de enfrentamientos generados por la diversidad de creencias e ideologías, y capaz de reconocer la dignidad de cada persona y el respeto a sus creencias y formas de vida. De acuerdo con Amador, Mateos y Esteban (2017), el fomento de valores universales constituye un compromiso del proceso de educar que persigue la construcción de una sociedad común que respete las diversas identidades.

Conviene no olvidar que si queremos partir desde un análisis certero de las realidades sociales tenemos que considerar que en muchos casos la principal acción educativa va a consistir en neutralizar contravalores o compensar carencias derivadas de la influencia de algunos 
de los agentes de socialización -especialmente la familia, el grupo de iguales, los medios de comunicación, etc.-, como, por ejemplo, los estereotipos y prejuicios trasmitidos por los medios de comunicación, la creación de mitos violentos por determinados agentes sociales, etc., aunque tenemos que aprender también a identificar y valorar las situaciones pacíficas y los comportamientos conciliadores presentes en dichos ámbitos de socialización.

Para conseguirlo hay que establecer mecanismos de diálogo y negociación con todos los agentes socioeducativos con el fin de llegar a establecer consensos para la creación de un clima escolar que facilite vivir situaciones de Cultura de Paz en los centros, para lo que resulta decisiva la implicación de dichos agentes y la participación de la comunidad educativa en todo el proceso de diálogo, negociación y establecimiento de normas de convivencia, que es imposible sin una democratización de los estilos de organización y gestión de los centros educativos.

Uno de los errores clásicos que se han venido dando al abordar educativamente los valores y las temáticas asociadas, como la Cultura de Paz y la interculturalidad, ha sido no valorarlas suficientemente como estilo de vida, como cultura institucional que dé sentido a las actuaciones que se realicen en los centros educativos (Sánchez, 2018). Tenemos que intentar aprovechar la vida -con sus manifestaciones de convivencia y sus conflictos- que tiene lugar en las escuelas, los institutos y las universidades para aprender de sus experiencias e igualmente intentar que sus estructuras organizativas y burocráticas se alejen de posturas discriminatorias y excluyentes.

Si las desarrollamos solamente en forma de contenidos educativos para su tratamiento en las aulas, sin considerarla en el ambiente del centro y en su vida diaria, caeremos en el error de olvidar la importancia que el aprendizaje por imitación y modelado tiene para cualquier persona, pero especialmente para los niños y jóvenes. Podemos estructurar buenos programas de contenidos y actividades educativas sobre el respeto a la diversidad para trabajarlas el aula, pero si las estructuras organizativas y de gestión del centro son autoritarias, poco participativas y contrarias a la convivencia, de nada habrán servidos aquellos, ya que nuestro alumnado aprenderá, sobre todo, de las experiencias que asimilen del estilo de vida y del ambiente del centro y menos de las tareas de aula, asociadas habitualmente a aprendizajes estrictamente académicos.

En cuanto a las relaciones con las comunidades educativas, podemos apreciar que, aunque se valore positivamente el respeto a la diversidad cultural, no se fomenta con la misma evidencia en algunas situaciones en las que se da una relación de cierta hipocresía hacia la escuela cuando al producirse fenómenos llamativos de violencia social -no necesariamente escolar-, determinados agentes sociales se 
vuelven hacia las instituciones educativas para reclamar la importancia de la educación en los asuntos relacionados con la prevención de la violencia. Incluso si algunos de los conflictos tienen lugar cerca de los centros escolares, se les asocia a éstos como causantes de los mismos. Así, resulta habitual denominar, incluso en estudios e investigaciones sobre el tema, como "escolar" a episodios de racismo, de xenofobia y de violencia que tienen lugar en espacios ajenos a los escolares sólo porque alguno de los agresores esté en edad escolar. Digamos que, sin negar que la sociedad reconoce y valora la importancia que tienen la educación y los centros escolares para prevenir estas conductas (Sánchez y Rojas, 2009), también tenemos que reconocer que en ciertas ocasiones se cae en una culpabilización recurrente de estas instituciones, que resulta injusta en la mayoría de los casos.

A continuación, se recogen las estrategias más habituales utilizadas para la gestión de los conflictos en los espacios y ámbitos educativos, que en la mayoría de los casos se combinan en su desarrollo práctico:

- La resolución, en la que prevalece la intervención externa a los implicados en el conflicto. Por ejemplo, un adulto (profesor, familiar, ...) en un conflicto entre niños; o un experto (juez, mediador, ...) en uno entre adultos. En las prácticas educativas, la intervención del profesorado en una relación conflictiva entre alumnos va a depender en gran medida de su preparación y de la capacidad de éstos para desarrollar por sí mismos la siguiente estrategia.

- La regulación o autorregulación, donde predomina la actuación directa de los afectados por el conflicto, asumiendo sus responsabilidades en todo el proceso de gestión. El papel de los agentes externos, si lo hubiera, es más sutil, concentrándose fundamentalmente en tareas de mediación, asesoramiento, observación y acompañamiento, debiendo quedar su intervención en un segundo plano. En los espacios escolares es frecuente que los propios niños inicien, en la mayoría de los casos, la autorregulación de un conflicto, especialmente si han tenido experiencias formativas previas.

- La transformación, marcada por la búsqueda y realización de cambios de escenarios, ambientes y protagonistas influyentes e implicados en el conflicto. Los agentes externos son fundamentales para asesorar las propuestas y el desarrollo de los cambios que se hagan, así como para buscar y favorecer los consensos necesarios al respecto, sobre todo, entre los afectados por el conflicto. Un ejemplo frecuente en los escenarios educativos es el cambio de compañeros, de clase o incluso de colegio.

Quedan por añadir la provocación "controlada" de los conflictos para aprender a gestionarlos en situaciones reales vividas en primera per- 
sona, y la evasión con tácticas esquivas para evitarlos con la intención de abordar posteriormente su gestión o para intentar olvidarlos, siendo ésta la opción menos recomendable desde el punto de vista educativo.

La mediación es una de las herramientas pedagógicas por excelencia para la prevención y gestión de los conflictos, basada en la confidencialidad, la objetividad y la neutralidad de la persona que media, como figura que facilita el diálogo, la toma ajustada de decisiones, la comunicación y la participación entre las partes, siendo consciente de que su poder se ejerce sobre el procedimiento en la resolución de conflictos, no así sobre las partes implicadas (Pérez Serrano y Pérez de Guzmán, 2011; Pérez de Guzmán, Amador Muñoz y Vargas Vergara, 2011).

Por todo ello, es necesario aprender a identificar y valorar las situaciones pacíficas y los comportamientos conciliadores presentes en todos los ámbitos de socialización, empezando por la creación en los espacios educativos de un ambiente y una cultura escolar favorables a la adquisición y al tratamiento de los valores de la interculturalidad en los centros y en las aulas. Para conseguirlo hay que establecer mecanismos de diálogo y negociación con otros agentes educativos con el fin de llegar a establecer consensos para la creación de un clima escolar que facilite vivir situaciones de paz, con sus imperfecciones y sus riquezas, en los centros.
Para ello, va a ser decisiva la implicación de dichos agentes y la participación de las comunidades educativas en todo el proceso de diálogo, negociación y establecimiento de normas de convivencia (Carretero, López y Sánchez, 2009). La educación para la paz debe utilizar el diálogo, el respecto y la cooperación como métodos básicos para poder comprender la complejidad de nuestra sociedad, prevenir y resolver conflictos.

\section{REFERENCIAS BIBLIOGRÁFICAS}

Amador Muñoz, L. V., Mateos Claros, F. y Esteban Ibáñez, M. (2017). La educación como medio para la inclusión social entre culturas (los valores sociales de los jóvenes ceutís de cultura cristiana y musulmana). Pedagogía social. Revista interuniversitaria, 29, pp. 67-80.

Binaburo, J. A. \& Muñoz, B. (2007). Educar desde el conflicto. Guía para la mediación escolar. Barcelona: CEAC.

Borlaug, N., Pérez Esquivel, A.; Dalai Lama, Gorbachov, M.S., Maguire, M., Mandela, N. et ál. (1999). Manifiesto 2000. París: Unesco. Recuperado de: http://www3. unesco.org/manifesto2000/sp/sp_manifeste.html

Carmona, $\mathrm{M}^{\mathrm{a}} \mathrm{J}$. (2016). Los niños que sueñan con quemar fronteras. Tinta Libre, 39, 2629.

Carretero, A., López, S. \& Sánchez, S. (2009). 
Vivir convivir. La convivencia escolar en centros de enseñanza secundaria desde una perspectiva intercultural. Sevilla: Federación Andalucía Acoge.

Gobierno de Colombia. Carta de Colombia. XVICumbre Mundial de Premios Nobeles de la Paz. Recuperado de http://es.presidencia.gov.co/noticia/170215-The-Colombia-Charter.

Lederach, J. P. (2003). Conflict transformation. Pennsylvania, USA: Good Books.

Mazorra, P. (2008). El conflicto en la escuela: Una oportunidad de aprendizaje. Bordón, 60 (4), 125-136.

Molina, B. \& Muñoz, F.A. (Eds.), (2004). Manual de Paz y Conflictos. Granada: Universidad de Granada y Consejería de Educación y Ciencia de la Junta de Andalucía.

Muñoz, F.A. (Ed.), (2001). La paz imperfecta. Granada: Universidad de Granada, colección Eirene.

Muñoz, F. A. (2004). Qué son los conflictos. En B. Molina \& F. A. Muñoz (Eds.), Manual de Paz y Conflictos (pp. 145-170). Granada: Universidad de Granada y Consejería de Educación de la Junta de Andalucía.

Pérez Serrano, G. y Pérez de Guzmán Puya, M.V. (2011). Aprender a convivir. El conflicto como oportunidad de crecimiento. Madrid. Narcea.

Pérez de Guzmán Puya, M.V., Amador Muñoz, L.V. y Vargas Vergara, M. (2011).
Resolución de conflictos en las aulas: un análisis desde la investigación-acción. Pedagogía Social. Revista Interuniversitaria, 18, pp. 99-114.

Ruiz, R. (Dir.) (2015). El tercer sector de acción social en 2015: Impacto de la crisis. Madrid: Plataforma de ONG de Acción Social y Plataforma Tercer Sector.

Sánchez, S. (2017). Las manifestaciones de la Cultura de Paz como fuente de contenido en la Educación para la Paz. Convives, 18, 6-11.

Sánchez, S. (2018). Respuestas educativas a la diversidad cultural. Una visión desde la perspectiva de la Cultura de Paz. Granada: Secretaría General de la Universidad de Granada.

Sánchez, S. \& Rojas, G. (2009). Peace Culture as Preventive Education Estrategy. En D. Checa (Ed.), Studying and Preventing the Radicalization of Is/am in Eastern Europe (pp. 70-84). Sofia-Granada: Universidad de Granada.

Sánchez-Vázquez, L. (2014). Mediación en conflictos ambientales. Una aproximación preliminar desde la Investigación para la Paz. Complexus, saberes entretejidos, 3, 11-15.

Segura, A. Alemany, I. \& Gallardo, M. A. (2016). Las actitudes prejuiciosas del alumnado universitario hacia los inmigrantes en situación irregular: Un estudio exploratorio. Electronic Journal of Research in 
Educational Psychology, 14(2) (39), 393416.

Segura, P. (2010). Pobreza y exclusión social. Diagnóstico de los distritos 4 y 5 de Melilla. Melilla: Acción Social sin Fronteras.

Trifu, L. A. (2018). Reflexiones sobre la paz positiva. Un diálogo con la paz imperfecta. En Revista de paz y conflictos, 11 (1), pp. 29-59.

UNESCO (1996). From a culture of violence to a culture of peace. París: Ediciones UNESCO.

Naciones Unidas (ONU) (2015). Objetivos de Desarrollo Sostenible. Recuperado de https://sustainabledevelopment.un.org/ sdgs

\section{PARA CITAR ESTE ARTÍCULO:}

Sánchez, S., Pérez, V., Rebolledo., y Rodríguez, R. (2019). La cultura de paz y conflictos: implicaciones socioeducativas. Collectivus, Revista de Ciencias Sociales, 6(1), 235-250. 